\title{
ARTICLE
}

\section{Acute myeloid leukemia}

\section{Chromothripsis in acute myeloid leukemia: biological features and impact on survival}

\author{
Maria Chiara Fontana ${ }^{1} \cdot$ Giovanni Marconi $\mathbb{B}^{1}$ - Jelena D. Milosevic Feenstra ${ }^{2}$ - Eugenio Fonzi ${ }^{1}$. \\ Cristina Papayannidis ${ }^{1}$ Andrea Ghelli Luserna di Rorá ${ }^{1} \cdot$ Antonella Padella $^{1} \cdot$ Vincenza Solli $^{1} \cdot$ Eugenia Franchini $^{1}$. \\ Emanuela Ottaviani ${ }^{1} \cdot$ Anna Ferrari $^{1} \cdot$ Carmen Baldazzi $^{1}{ }^{1} \cdot$ Nicoletta Testoni $^{1} \cdot$ Ilaria lacobucci ${ }^{1,3}$. \\ Simona Soverini ${ }^{1} \cdot$ Torsten Haferlach $^{4} \cdot$ Viviana Guadagnuolo $^{1} \cdot$ Lukas Semerad $^{5}$ - Michael Doubek ${ }^{5}$. \\ Michael Steurer ${ }^{6} \cdot$ Zdenek Racil $^{5} \cdot$ Stefania Paolini ${ }^{1} \cdot$ Marco Manfrini ${ }^{1} \cdot$ Michele Cavo $^{1} \cdot$ Giorgia Simonetti $^{1}$. \\ Robert Kralovics ${ }^{2} \cdot$ Giovanni Martinelli $^{1}$
}

Received: 4 August 2017 / Revised: 31 October 2017 / Accepted: 21 November 2017 / Published online: 23 February 2018

(c) The Author(s) 2018. This article is published with open access

\begin{abstract}
Chromothripsis is a one-step genome-shattering catastrophe resulting from disruption of one or few chromosomes in multiple fragments and consequent random rejoining and repair. This study defines incidence of chromothripsis in 395 newly diagnosed adult acute myeloid leukemia (AML) patients from three institutions, its impact on survival and its genomic background. SNP 6.0 or CytoscanHD Array (Affymetrix ${ }^{\circledR}$ ) were performed on all samples. We detected chromothripsis with a custom algorithm in 26/395 patients. Patients harboring chromothripsis had higher age $(p=0.002)$, ELN high risk (HR) $(p$ $<0.001)$, lower white blood cell (WBC) count $(p=0.040)$, TP53 loss, and/or mutations $(p<0.001)$ while FLT3 $(p=0.025)$, and NPM1 $(p=0.032)$ mutations were mutually exclusive with chromothripsis. Chromothripsis-positive patients showed a worse overall survival (OS) $(p<0.001)$ compared with HR patients $(p=0.011)$ and a poor prognosis in a COX-HR optimal regression model. Chromothripsis presented the hallmarks of chromosome instability [i.e., TP53 alteration, 5q deletion, higher mean of copy number alteration (CNA), complex karyotype, alterations in DNA repair, and cell cycle] and focal deletions on chromosomes 4, 7, 12, 16, and 17. CBA. FISH showed that chromothripsis is associated with marker, derivative, and ring chromosomes. In conclusion, chromothripsis frequently occurs in AML (6.6\%) and influences patient prognosis and disease biology.
\end{abstract}

These authors contributed equally: Maria Chiara Fontana, Giovanni Marconi.

These authors jointly supervised this work: Giorgia Simonetti, Robert Kralovics, Giovanni Martinelli.

Electronic supplementary material The online version of this article (https://doi.org/10.1038/s41375-018-0035-y) contains supplementary material, which is available to authorized users.

Maria Chiara Fontana

mariachiara.fontana4@unibo.it

$\triangle$ Giovanni Marconi

giovanni.marconi@studio.unibo.it

$\bowtie$ Giovanni Martinelli

giovanni.martinelli2@unibo.it

1 Institute of Hematology "L. and A. Seràgnoli", University of Bologna, Bologna, Italy

\section{Introduction}

Loss of chromosomes and complex karyotype are mechanisms of genomic instability known to be linked to therapy resistance, poor prognosis, and early relapse in AML [1-3]. Nowadays, new high-throughput technologies can discover new alterations responsible for the poor prognosis in subcohorts of AML patients and may reveal

2 CeMM Research Center for Molecular Medicine of the Austrian Academy of Sciences, Wien, Austria

3 Department of Pathology, St. Jude Children's Research Hospital, Memphis, TN, USA

4 MLL Munich Leukemia Laboratory, Munich, Germany

5 Department of Internal Medicine - Hematology and Oncology, Masaryk University and Hospital, Brno, Czech Republic

6 Present address: Division of Hematology and Oncology, Medical University of Innsbruck, Innsbruck, Austria 
druggable pathways. There is an urgent need to define genomic phenotypes in AML in a therapeutic perspective.

Chromothripsis is a one-step genomic catastrophe involving one or few chromosomes resulting from chromosome breakage and random DNA rejoining [4]. It has been detected, mainly as occasional finding, in solid tumors [5-13] and hematological malignancies (multiple myeloma [14], AML [15, 16], acute lymphoblastic leukemia [17, 18], and chronic lymphocytic leukemia [19]), as well as in germline cases of congenital disorders [20]. A study on 22,000 cases of primary tumors highlighted an overall incidence of chromothripsis between 2 and 3\% [21].

During mitosis, chromothripsis arises from aberrant DNA replication timing. Prolonged arrest of cell cycle and micronuclei formation influence the spatial distribution of damages favoring the acquisition of structural rearrangements [22]. Molecular mechanisms implicated in this event are only partially discovered. Chaotic genomes seemed to form through random non-homologous end joining after DNA damages [23]. An alternative theory presented chromothripsis as a putative incomplete outcome of chromosome fragmentation, the initial event triggering a new form of mitotic cell death [23]. Moreover, recent evidence indicates that chromothripsis preferentially occurs especially in hyperploid cells [24] and in cells with damaged telomeres [25]. Chromothripsis has been associated to EGFR, MDM2, and MDM4 amplification, CDKN2A and PTEN deletion [8], aberrant DNA double-strand break (DSB) response [26], TP53 mutations [9, 15], complex karyotype [9, 15], and ATM mutation [18].

At present, a standardized way to detect chromothripsis does not exist, but few have been proposed. Chromothripsis has been identified with fluorescent in situ hybridization (FISH) $[9,15,16,26]$, or, alternatively, by operatordependent analyses [14] as well as algorithm-based detection of shattering patterns on SNP array data and eventual integration with DNA sequencing data [21, 27]. Korbel and Campbell defined six different criteria to distinguish chromothripsis from a multi-step process of genomic rearrangement [28]. When analyzed by SNP arrays, chromosomes harboring chromothripsis show a characteristic pattern of alterations: two or three switches of $\mathrm{CN}$ state detectable along the chromosome with a clustering of breakpoint locations [29]. These patterns are associated with a high number of chromosomal rearrangements with widespread loss or gain of sequence fragments interspersed in diploid regions [21].

Chromothripsis has been associated with highly aggressive disease in various tumors [8-14] but it did not appear to impact prognosis in prostate cancer [30] and in estrogen receptor-negative breast cancer [12].

Our study describes the incidence of chromothripsis at diagnosis in a large and homogenous AML cohort, its impact on prognosis, and the genetic features associated with this phenomenon.

\section{Subjects and methods}

\section{Patients}

Samples and data at diagnosis from 395 adult patients affected by de novo or secondary AML according to WHO 2016 criteria [31] were collected from three institutions. The study was approved by the local ethical committees, written permission, and informed consent were obtained from all patients before sample collection according to Helsinki declaration of "Ethical Principles for Medical Research Involving Human Subjects" [32].

Data were collected and managed through custom Electronic Case Report Forms using REDCap electronic data capture tool [33].

\section{SNP microarray analysis}

DNA samples were processed by Affymetrix ${ }^{\circledR}$ (Santa Clara, CA, USA) genome-wide human SNP $6.0(n=321)$ and CytoscanHD Array $(n=84)$ according to the manufacturer's instructions. Of the 114 SNP 6.0 AML cases obtained from GSE23452, 112 were paired samples, including buccal swab DNA or bone marrow at remission [34]. Array data have been deposited in the NGS-PTL repository (http://www.ngs-ptl.com/documents/documents/ 3-10-en/media.aspx).

\section{Detection of chromothripsis}

Chromothripsis was defined according to Korbel and Campbell's criteria [28], when three out of six criteria were satisfied (the remaining criteria could not be assessed by SNP array analysis).

Chromothripsis was assessed by scanning SNP array segment files using CTLP-Scanner (using R v3.3.2 [35] and "CTLP scanner" package [21]). The following thresholds were set: $\log$ ratio $\geq 8$, more than 10 breakpoints, minimum segment size of $10 \mathrm{~kb}$, and 0.3 as signal distance between adjacent segments. Events with a prevalent copy number $(\mathrm{CN})$ status and changes involving $\leq 10 \%$ of detected region were excluded.

\section{Microarray statistical analyses}

Based on the presence/absence of chromothripsis, AML samples were divided in two groups: a group of cases (chromothripsis-positive) and a group of controls (chromothripsis-negative) and enrichment of CNA events 
between the two groups was examined. The data set was stratified for event type and the statistical tests were performed on amplifications of one or more DNA copies and heterozygous or homozygous deletions. In each patient, multiple events of the same type in the same gene were considered as one. Fisher's exact test was used to compare frequencies in genes' event between two groups. All $p$ values were adjusted for multiple testing. For testing at a pathway level, genes were annotated in the Reactome database [36]. Pathway enrichment analysis was performed at patient level by means of an over-representation test. Then, the adjusted $p$ values obtained for a certain pathway across all patients were used as predictor variable in a logistic regression model. The significance level was set at $10^{-4}$.

\section{Chromosome banding analysis and FISH}

FISH analysis was carried out on previously G-banded metaphases prepared by chromosome banding analysis (CBA) technique according to the manufacturer's instructions.

\section{Clinical statistical analysis}

Due to the data-pool feature of our set, missing data will be detailed in the result section. OS was assessed as the time in days from diagnosis to death or last follow-up.

Fisher's exact test and chi-squared test were used for crosstabs and difference between distributions was assessed with median test for independent samples and Mann-Whitney $U$ test. Survival analysis and COX-HR were used as appropriate.

\section{Results}

\section{Clinical and molecular patient characteristics}

All patients' clinical and molecular characteristics are listed in Table 1, missing data are quantified in Table S1. In our cohort, 26 out of 395 patients $(6.6 \%)$ showed chromothripsis involving different chromosomes.

\section{Correlation of chromothripsis with clinical and molecular parameters in AML patients}

We compared chromothripsis-negative patients with chromothripsis-positive ones (Table 2). Chromothripsispositive patients were older. They had a higher median age (67 and 60 years, respectively $p=0.002$, Figure S1C) and a lower WBC count mean at diagnosis $\left(6342 / \mathrm{mm}^{3}\right.$ vs. $30,059 /$ $\mathrm{mm}^{3}$, respectively $p=0.040$, Figure S1A). Chromothripsis-
Table 1 Clinical and molecular characteristics of the patients enrolled in this study $(N=395)$

\begin{tabular}{|c|c|}
\hline Parameter & Value \\
\hline Age & $\begin{array}{l}\text { Median } 59.35 \text { (range, } \\
\text { 16-92) }\end{array}$ \\
\hline \multirow[t]{2}{*}{ Sex } & $\begin{array}{l}185 / 395 \text { patients were } \\
\text { female }(46.8 \%)\end{array}$ \\
\hline & $\begin{array}{l}210 / 395 \text { patients were male } \\
(53.2 \%)\end{array}$ \\
\hline De novo AML & $307 / 372$ patients $(82.5 \%)$ \\
\hline $\begin{array}{l}\text { AML secondary to myelodysplastic } \\
\text { syndrome }\end{array}$ & $43 / 372$ patients $(11.6 \%)$ \\
\hline $\begin{array}{l}\text { AML secondary to other myeloid } \\
\text { neoplasms }\end{array}$ & $4 / 372$ patients $(1.1 \%)$ \\
\hline Therapy-related AML & $18 / 372$ patients $(4.8 \%)$ \\
\hline WBC (mean) & $\begin{array}{l}28.140 / \mathrm{mm}^{3}(100-171.00 / \\
\left.\mathrm{mm}^{3}\right)\end{array}$ \\
\hline WBC count greater than $30,000 / \mathrm{mm}^{3}$ & $46 / 154$ patients $(29.9 \%)$ \\
\hline WBC count greater than $100,000 / \mathrm{mm}^{3}$ & $12 / 154$ patients $(7.8 \%)$ \\
\hline $\begin{array}{l}\text { European LeukemiaNet (ELN) low } \\
\text { risk [37] }\end{array}$ & $35 / 352$ patients $(8.9 \%)$ \\
\hline $\begin{array}{l}\text { European LeukemiaNet (ELN) } \\
\text { intermediate } 1 \text { risk [37] }\end{array}$ & $100 / 352$ patients $(28.4 \%)$ \\
\hline $\begin{array}{l}\text { European LeukemiaNet (ELN) } \\
\text { intermediate } 2 \text { risk [37] }\end{array}$ & $80 / 352$ patients $(22.7 \%)$ \\
\hline $\begin{array}{l}\text { European LeukemiaNet (ELN) high } \\
\text { risk [37] }\end{array}$ & $137 / 352$ patients $(38.9 \%)$ \\
\hline Chemotherapy in induction & $251 / 308$ patients $(81.5 \%)$ \\
\hline $\begin{array}{l}\text { Gemtuzumab Ozagomicin was } \\
\text { added }\end{array}$ & $42 / 251$ patients $(16.7 \%)$ \\
\hline Complete remission & $153 / 251$ patients $(60.9 \%)$ \\
\hline Hematopoietic stem cell transplant & $85 / 283$ patients $(30.0 \%)$ \\
\hline $\begin{array}{l}\text { Loss or a mutation of TP53 at } \\
\text { diagnosis }\end{array}$ & $63 / 395$ patients $(15.9 \%)$ \\
\hline Loss & $29 / 395$ patients $(7.3 \%)$ \\
\hline Mutation & $53 / 324$ patients $(16.4 \%)$ \\
\hline Internal tandem duplication in $F L T 3$ & $42 / 298$ patients $(14.1 \%)$ \\
\hline $\begin{array}{l}\text { Tyrosine kinase domain mutation in } \\
\text { FLT3 }\end{array}$ & $18 / 298$ patients $(6.0 \%)$ \\
\hline Mutations in $N P M 1$ gene & $50 / 286$ patients $(17.5 \%)$ \\
\hline Mutations in $I D H I$ gene & $4 / 121$ patients $(3.3 \%)$ \\
\hline Mutations in $I D H 2$ gene & $11 / 135$ patients $(8.3 \%)$ \\
\hline Mutations in $D N M T 3 A$ gene & $7 / 38$ patients $(19.4 \%)$ \\
\hline Mutations in $C E B P A$ gene & $5 / 106$ patients $(4.7 \%)$ \\
\hline Mutations in $R U N X 1$ gene & $11 / 87$ patients $(12.6 \%)$ \\
\hline Mutations in $C B L$ gene & $2 / 91$ patients $(2.2 \%)$ \\
\hline Mutations in NRAS gene & $10 / 95$ patients $(10.5 \%)$ \\
\hline
\end{tabular}

positive patients presented a prevalence of complex karyotype and were classified as HR disease according to ELN [37] definition ( $p<0.001$, Figure S1D).

Based on genetic features, chromothripsis was associated with TP53 loss $(p<0.001)$ and TP53 mutations $(p<0.001)$. 


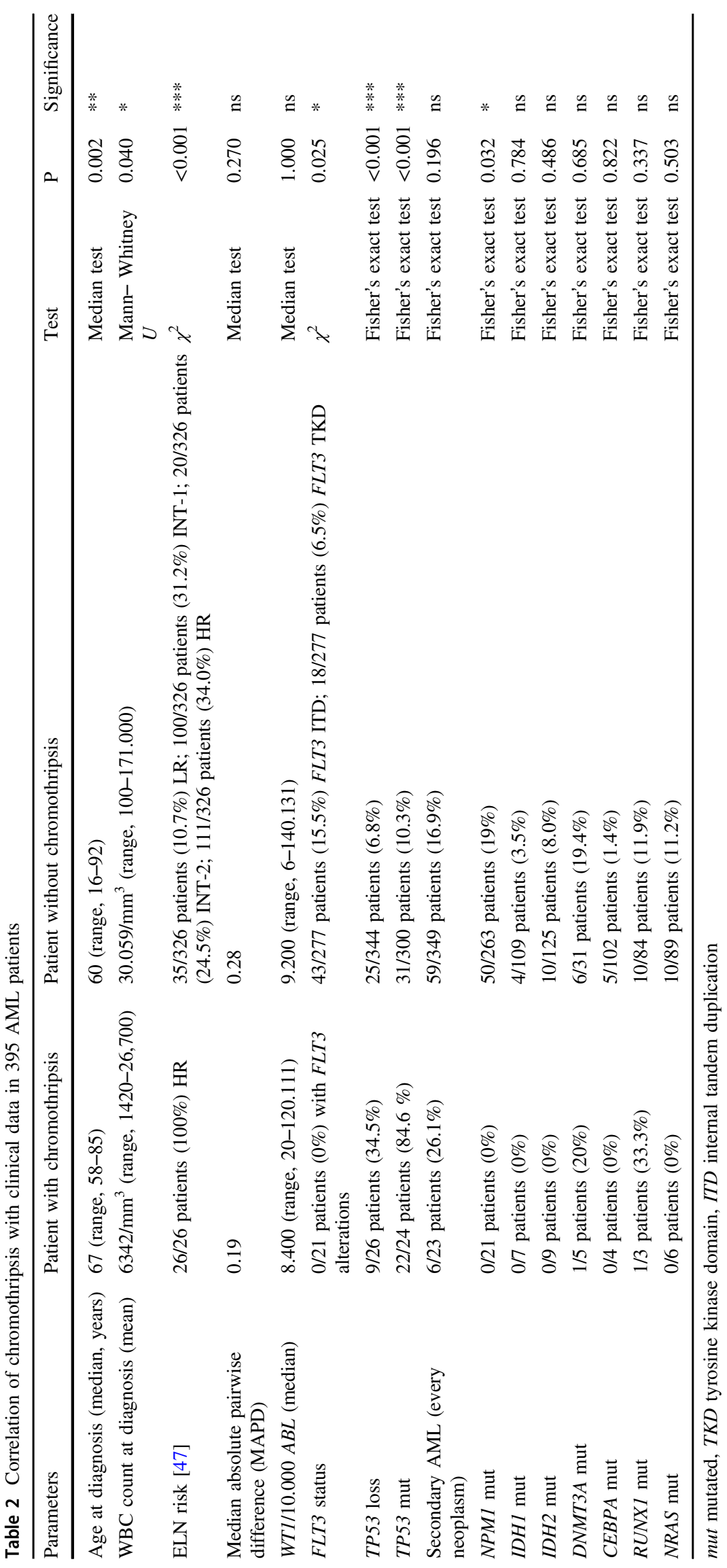


Table 3 Induction therapies in patients with and without chromothripsis

\begin{tabular}{lll}
\hline Induction therapy & \multicolumn{2}{l}{ Chromothripsis } \\
\cline { 2 - 3 } & No (288) & Yes (20) \\
\hline Chemotherapy-n. & 241 & 10 \\
Hypomethylating agents-n. & 23 & 4 \\
Best supportive therapy-n. & 24 & 6 \\
\hline
\end{tabular}

Only 3/26 patients did not harbor any TP53 alteration: one patient was TP53 wild-type and have no karyotype aberration involving $17 \mathrm{p}$, two patients were not tested for TP53 mutation because of unavailable material at diagnosis. Chromothripsis was mutually exclusive with FLT3 (Figure $\mathrm{S} 1 \mathrm{~B}, p=0.025)$ and NPM1 mutations $(p=0.032)$.

\section{Chromothripsis defined a group of AML patients with poor response to induction therapy}

At diagnosis, patients received chemotherapy, hypomethylating agents, or best supportive therapy (Table 3). Patients with chromothripsis were treated with intensive chemotherapy in a smaller proportion $(p=0.003)$. There was no difference in use of Gemtuzumab Ozagomicin during induction between the two group of patients $(p=1.000)$ and there was a similar transplant rate between the two groups [ $21 \%$ of patients with chromothripsis and $31 \%$ of patients without chromothripsis received hematopoietic stem cell transplant (HSCT), $p=0.448$ ].

Chromothripsis defined a group of patients with poor prognosis. Three out of 10 patients with chromothripsis (30\%) responded to induction, a significant lower proportion if compared with $152 / 229$ patients without chromothripsis $(66.4 \%, p=0.036)$.

\section{Chromothripsis defined a group of AML patients with poor OS}

Patients with chromothripsis showed a worse OS (median OS of 120 days compared to 494 days for patients without chromothripsis, $p<0.001$, Fig. 1a). In patients with available HSCT data, we confirmed the difference by censoring OS at HSCT with a median OS of 120 and 400 days in the two groups $(p<0.001$, Fig. 1b). Patients with chromothripsis had the worst prognosis among patients with HR features according ELN [37] risk stratification (median OS of 120 and 258 days in the two groups, $p=0.011$, Fig. 1c). This observation was confirmed when censoring OS at HSCT (median OS of 120 and 211 days in the two groups, $p=0.022$, Fig. 1d). Moreover, the impact of chromothripsis on OS was evaluated in patients with HR features according ELN [37] risk stratification, who received induction chemotherapy. We report a difference in survival between patients with and without chromothripsis (median OS of 120 and 295 days, respectively, $p=0.040$, Fig. 1e) and a trend toward statistical significance in HSCT-censored analysis (median OS of 120 and 242 days, respectively, $p$ $=0.055$, Fig. 1f). Patients with chromothripsis did not show differences in baseline characteristics or in survival compared to patients harboring TP53 mutation, TP53 loss, or to the group of patients harboring TP53 alteration (loss and/or mutation), due to the high co-occurrence of these two phenomena (Figure S2 and Table S2). However, in our set, patients with chromothripsis showed a survival disadvantage near to the statistical significance threshold when compared with patients with TP53 loss (Fig. S2B, $p=$ 0.049).

\section{Chromothripsis was an independent predictor of shorter OS in COX-HR model}

We built a prognostic model using COX-HR with forward conditional method, considering chromothripsis event, secondary AML, ELN risk, induction therapy, FLT3 and NPM1 mutation as categorical variables and age at diagnosis. TP53 status was not included in the model for the high co-occurrence of chromothripsis and TP53 alterations. In the optimal model, chromothripsis (HR: $2.286,95 \% \mathrm{CI}$ : $1.327-3.940, p=0.003$ ) and secondary disease was associated with augmented risk of death, while ELN low risk, intermediate 1 and intermediate 2 risk were associated with better outcome (Fig. 2). Chromothripsis was a consistent risk factor in COX-HR model built in ELN HR population considering chromothripsis, secondary AML, induction therapy, FLT3 mutation, and NPM1 mutation as categorical variables, and age of diagnosis (HR: 2.070, 95\% CI: 1.167-3.672, $p=0.013$, model not shown). Chromothripsis was also a consistent risk factor in COX-HR model built in ELN HR population treated with intensive therapies considering chromothripsis, secondary AML, FLT3 mutation, and NPM1 mutation as categorical variables, and age of diagnosis (HR: 2.227, 95\% CI: 1.022-4.850, $p=0.044$, model not shown).

\section{Genomic characteristics of AML patients with chromothripsis}

We detected chromothripsis on chromosome 12 (23\% of events), 17 and 5 (17\% of events both), chromosomes 6 (10\% of events), 3 and 8 (6.6\% of events both), 7, 10, 11, 15, and 20 (3.3\% of events each) (Fig. 3, and Fig. S3). SNP array analyses showed that chromothripsis-positive patients were characterized by a high grade of genomic aberrations.

We found a minimal common-deleted region in 24/26 patients with chromothripsis (5q31.1-5q33.1). Among 
(A)

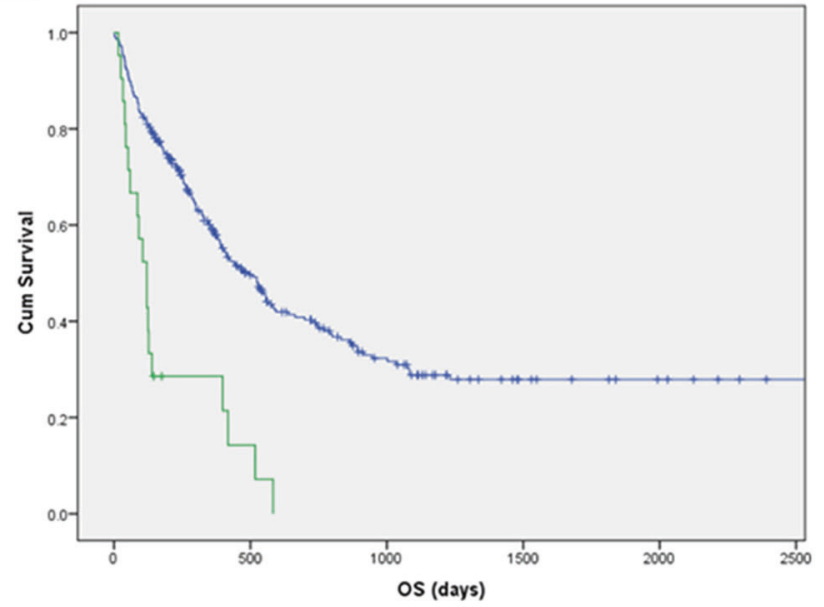

(C)

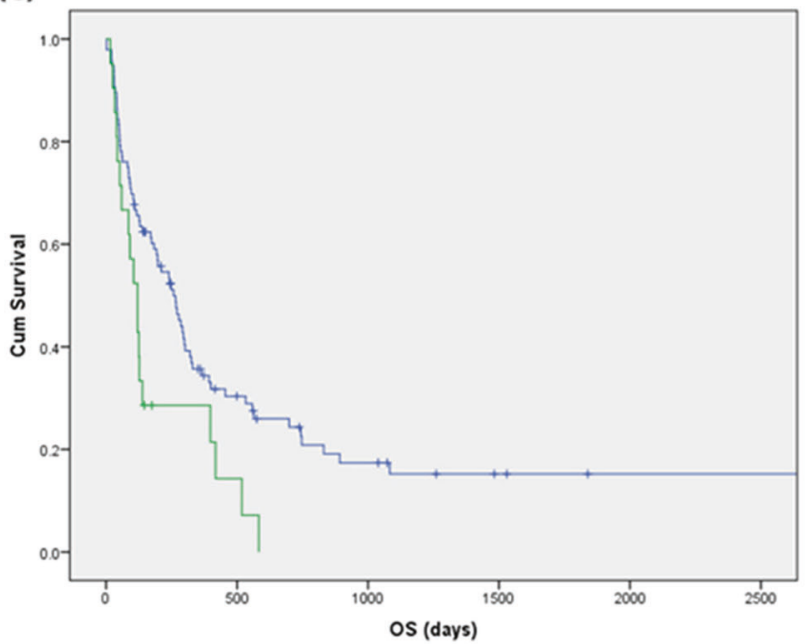

(E)

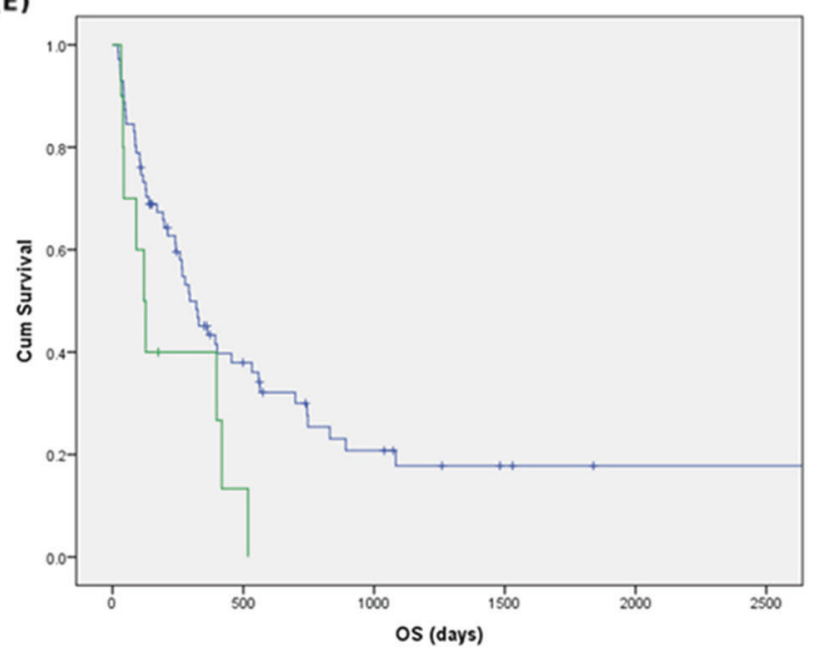

Fig. 1 Association between chromothripsis and OS in AML. OS in patients with (green line) and without chromothripsis (blue line): a overall cohort; b survival censored at HSCT; c patients with ELN [37] HR features; d patients with ELN [37] HR features censoring survival
(B)

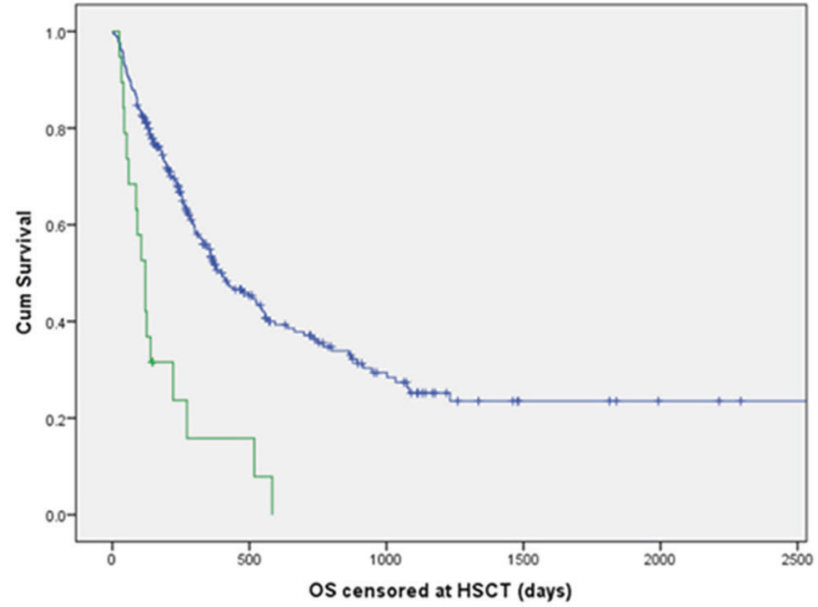

(D)

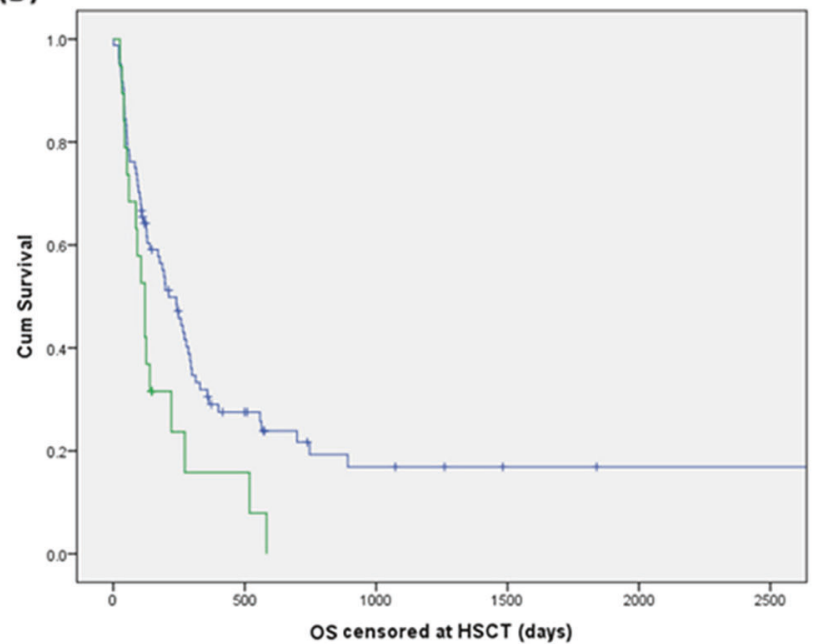

(F)

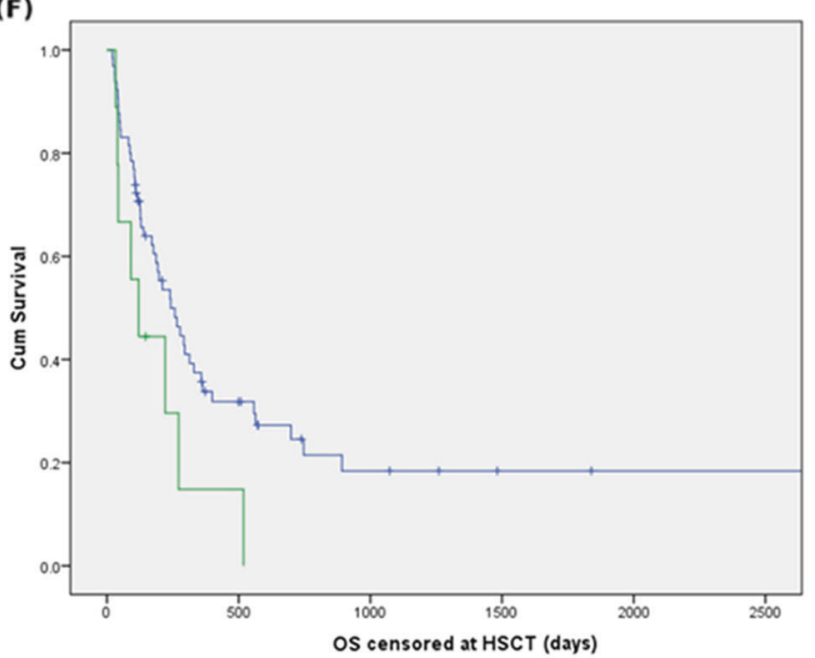

at HSCT; e patients treated with intensive chemotherapy with ELN $2017 \mathrm{~h}$ features; f patients treated with intensive chemotherapy with ELN [37] HR features censoring survival at HSCT 
(A)

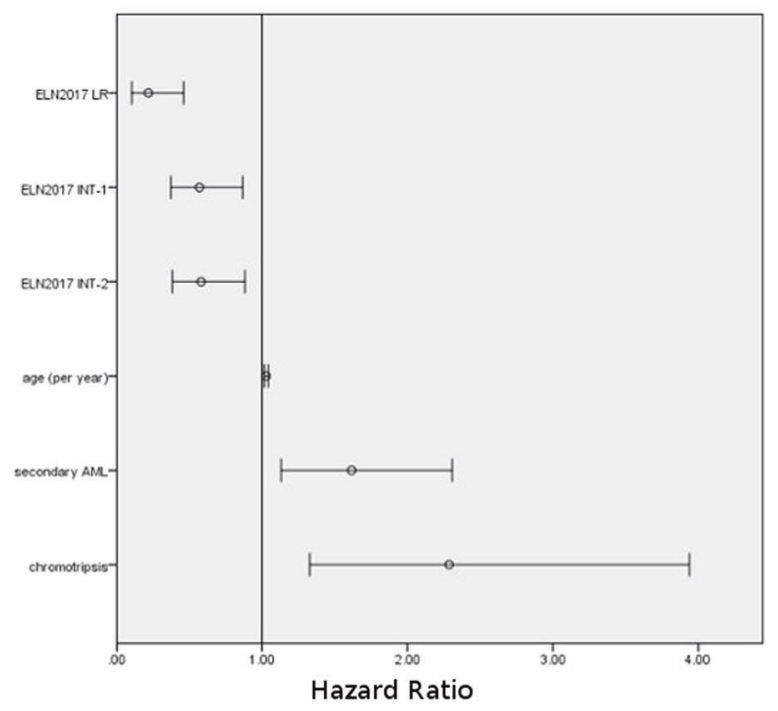

(B)

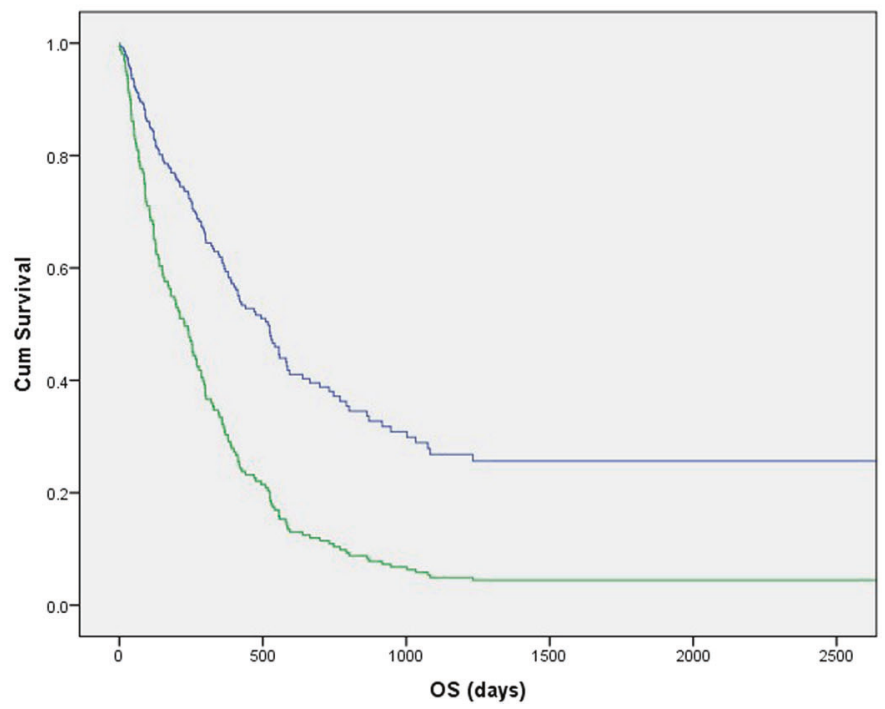

Fig. 2 COX-HR model. COX-HR model in patients' set considering (a) forest plot of risk factors in COX-HR optimal model (b) in COX-HR model, difference in OS (OS) representing population with (green line) and without (blue line) chromothripsis

chromothripsis-negative patients, 51/369 harbored at least a $\mathrm{CNA}$ of $9 \mathrm{Mb}$ in the $5 \mathrm{q}$ arm. There was a higher incidence in macroscopic deletions on $5 \mathrm{q}$ in patients with chromothripsis $(p<0.0001)$.

Patients with chromothripsis presented higher mean of CNA than patients without chromothripsis (mean of 418 vs. 188 CNA per patient; Fig. 4, CIRCOS [38] external level).

\section{Significative genomic alterations mapped in relatively small chromosomic regions}

Fisher's exact test showed that a large group of 1359 genes were significantly altered in deletion (both heterozygous and homozygous) in chromothripsis-positive patients rather than chromothripsis-negative ones (data not shown). These genes map on chromosome 5q, 3q, 12p, 3p, 4q, 7q, 12p, $16 \mathrm{q}$, and $17 \mathrm{p}$. Considering chromosome position of genes associated with chromothripsis, we found that CNA randomly affected the entire $5 \mathrm{q}$ and whole chromosome 3 . In the other chromosomes, we found that statistically significant CNA mapped in relatively small regions (complete list of genes in Fig. 4, CIRCOS [38] internal level). These regions included deletions of genes involved in Atlas of Genetics and Cytogenetics in Oncology and Haematology, in particular on chromosome 4q28-32 (SFRP2), 7q31.1-36.3 (CAV1, EPHA1, and NRF1), 12p11.21-13.3 (EPS8, RECQL, and GUCY2C), 16q22-24.3 (transcription factors CBFA2T3 and FOXF1; CDT1 involved in DNA replication; and the Fanconi Anemia gene FANCA), and 17p13-13.1 (ALOX12 and CLDN7) (Fig. 4). Genes were filtered as described in "Methods" and we showed that 95 genes were associated with chromothripsis (complete list of genes in Fig. 4, CIRCOS [38] internal level).

\section{Pathway enrichment in patients with chromothripsis}

REACTOME enrichment of pathways is reported in Tables S3 and S4. DNA repair, E2F-mediated regulation of DNA replication, signaling pathways involving PI3K, phospholipid biosynthesis and metabolism, and various growth factors signaling pathways scored in the best $1 \%$ of pathways enriched for amplification events in chromothripsis. CTLA4 inhibitory signaling, synthesis of phosphatidylinositol phosphate (PIP) at the late endosome membrane, fanconi anemia pathway, genes regulating G0 and early G1 phase, pre-NOTCH transcription and translation scored in the best $1 \%$ of pathways enriched for deletion events in cases with chromothripsis.

In the subcohort of patients with TP53 alteration, when we compared patients with chromothripsis $(n=22)$ and patients without chromothripsis $(n=44)$, we did not detect any significant difference in terms of differentially altered genes and pathways (with a significance threshold of $p<$ $\left.10^{-4}\right)$.

\section{Chromothripsis is associated with marker, derivative, and ring chromosomes formation}

In order to better characterize the chromosomes affected by chromothripsis, we performed FISH analysis in 7/26 cases with available material. Most cases (5/7) showed the 
a.

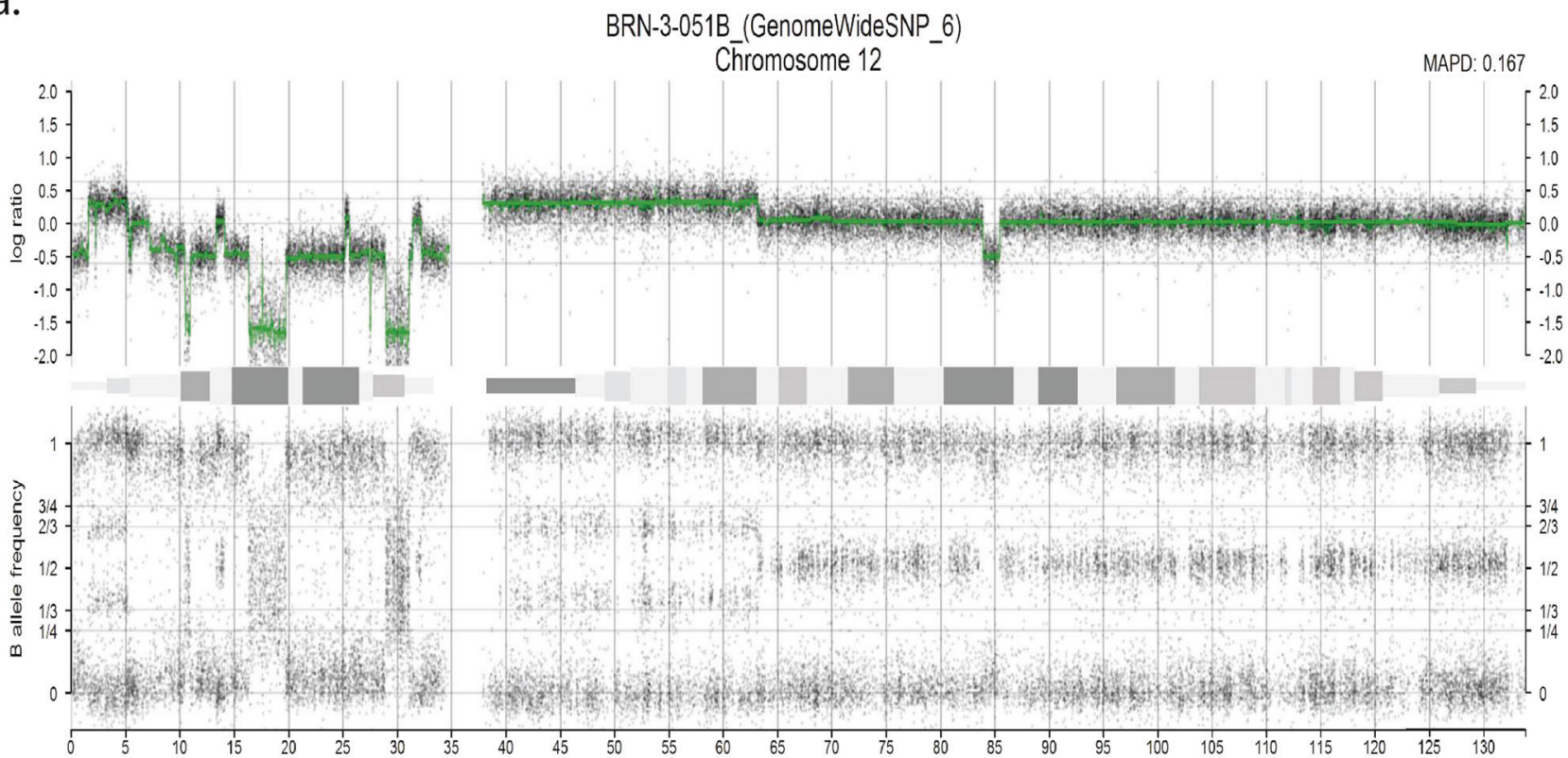

b.

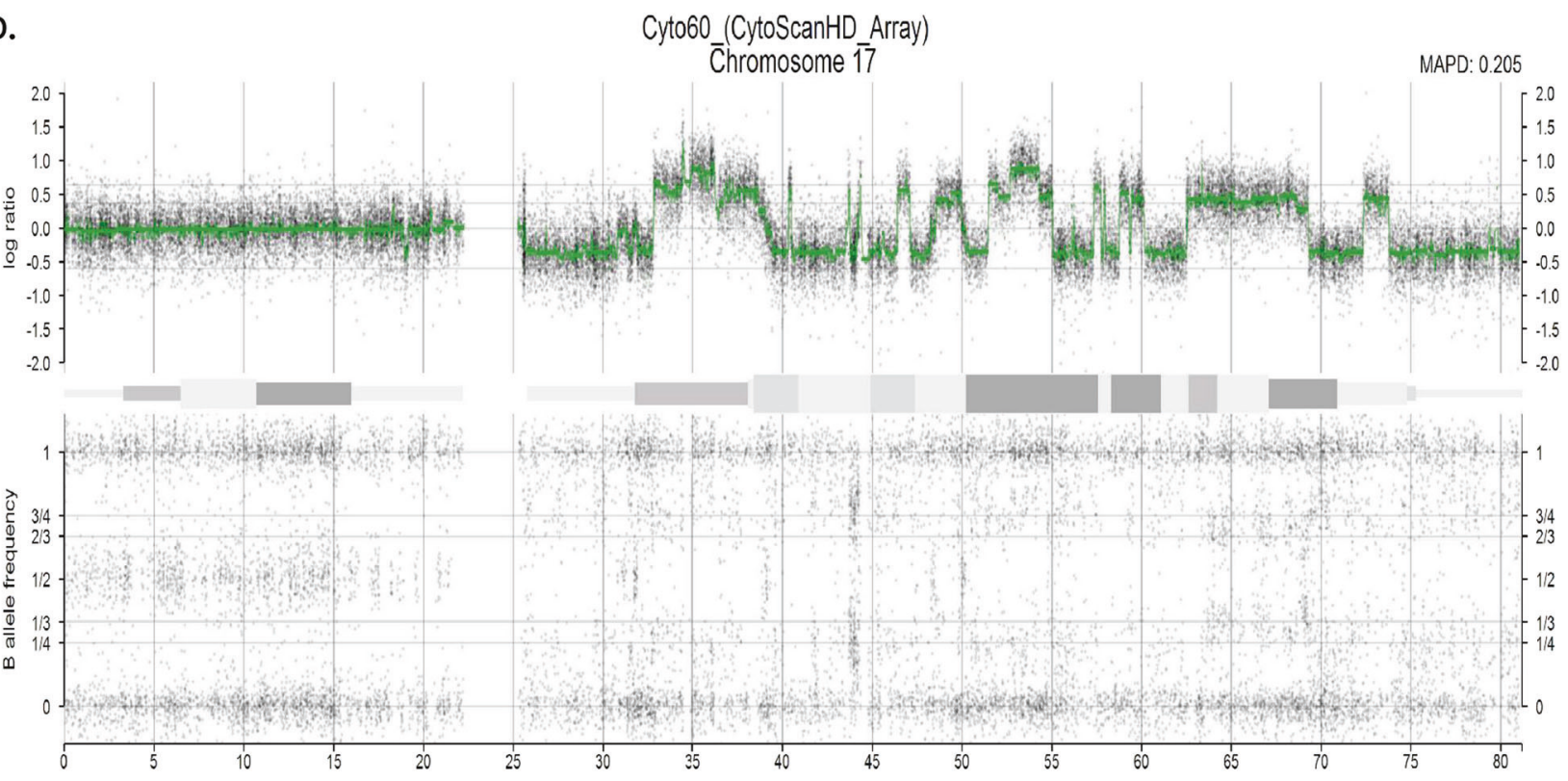

Fig. 3 Representation of two chromosomes affected by chromothripsis in different patients, plotted with Rawcopy v. 1.0. a Chromosome $17 \mathrm{q}$ with 36 switches and 2-3 changes in $\mathrm{CN}$ (involving also homozygous

gains). b Chromosome 12 p with 28 switches and 2-3 changes in $\mathrm{CN}$ (involving also homozygous losses)

presence of marker or ring chromosomes. In four cases, the chromosomes affected by chromothripsis (chromosomes 8 , 11,12 , and 17) were reported by CBA as monosomic chromosomes, while, by FISH, portions of these chromosomes were identified on marker chromosomes. In cases involving chromosomes 8 and 11, the rearrangement led to amplification of MYC and KMT2A (MLL) genes, respectively. In cases involving chromosomes 12 and 17, parts of the chromosomes affected by chromothripsis were identified on markers and on derivative chromosomes resulting from unbalanced translocations. In other two cases, the chromosome involved in chromothripsis was annotated as derivative chromosome. Moreover, FISH highlighted the presence of a homogeneously staining region on the derivative chromosome 3 due to MDS1 and EVI1 complex locus amplification in one case and of a complex translocation involving chromosome 5 in the other case. In the last patient, the chromosome affected by chromothripsis was identified as ring chromosome 17 leading to loss of TP53. FISH results are shown in Fig. 5. 


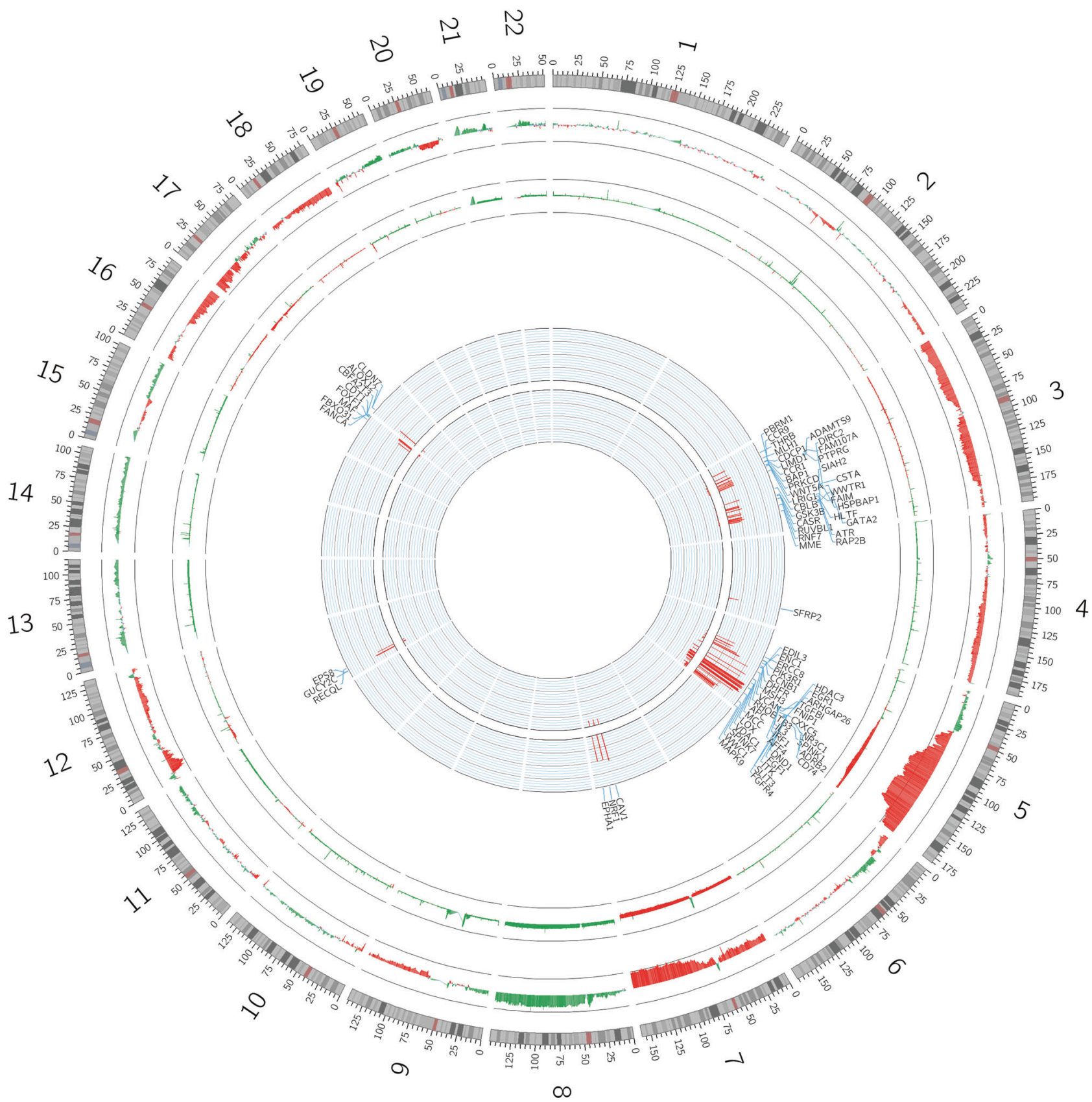

Fig. 4 CIRCOS plot representing the overall CNA and genes altered in the two groups (chromothripsis-positive patients vs. chromothripsisnegative patients). a External circular level: genomic landscape in CNA per group of patients (external line represents chromothripsispositive patients, internal line represents chromothripsis-negative patients). Green lines represent amplifications, red lines represent

deletions. b Internal list of selected genes (basing on Fisher's exact test and Atlas of Genetics and Cytogenetics in Oncology and Haematology) deleted in heterozygosis and/or homozygosis with relative frequency in CNA displayed with histograms: chromothripsis-positive patients (external level) and chromothripsis-negative patients (internal level)

\section{Discussion}

The purposes of this study was to define the incidence of chromothripsis in newly diagnosed adult AML patients, its impact on survival, and its genomic background in AML. To detect chromothripsis, we used a custom algorithm based on CTLP scanner [21] and Korbel and Campbell's

criteria [28] that detected chromothripsis based on SNP array data.

Our results, obtained in a large set of patients, indicate that chromothripsis is a non-anecdotal finding in AML. The overall incidence of chromothripsis was concordant with studies conducted on fewer patients $[15,16]$. Chromothripsis appeared to be associated with higher age and lower 


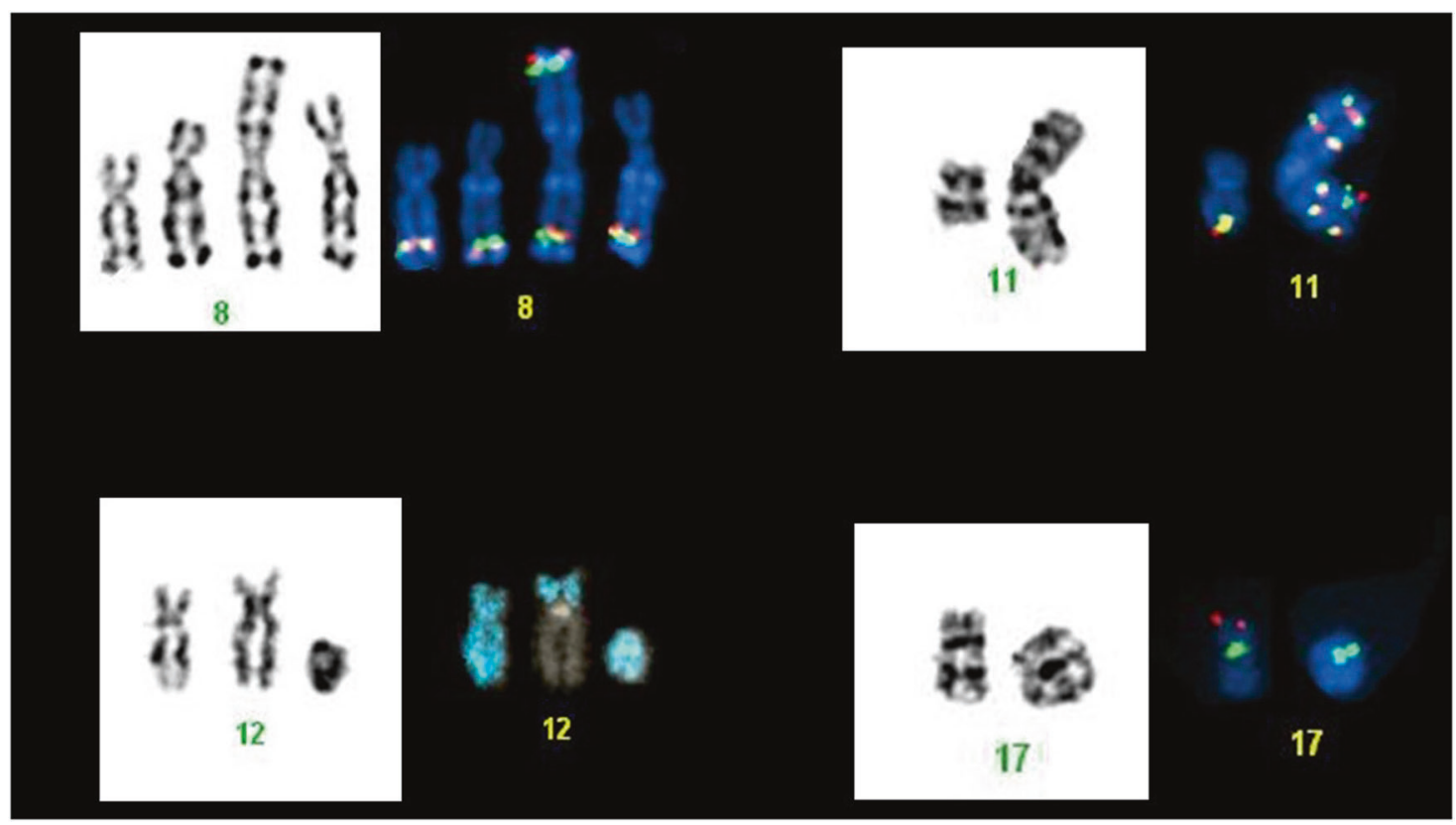

Fig. 5 Karyotype analysis in AML patients harboring chromothripsis. FISH analysis with a MYC breakapart probe showing five fusion signals of MYC localized on normal chromosome 8 and on three different marker chromosomes in patient with chromothripsis of chromosome 8. b KMT2A (MLL) breakapart probe showing four copies of KMT2A (four fusion signals) localized on the marker

WBC count at diagnosis, and mutually exclusive with FLT3 and NPM1 mutations, these data were never reported in acute leukemia. Moreover, we confirmed the strong association between chromothripsis and TP53 dysregulation [9, 15], thus reinforcing the importance of TP53 for the maintenance of genomic stability and integrity.

For the first time, we pointed out that the only detection of chromothripsis is sufficient to define a group of patients with poor prognosis in the general AML population and in the ELN [37] high-risk population; chromothripsis was a determinant of poor OS in the COX-HR optimal model. We further performed survival analysis in the subset of patients with TP53 alterations, as TP53 alone was reported to define patients with the worst prognosis in AML [39-41]; we did not detect differences in survival defined by to have or not to have chromothripsis, and this was the main limitation of our study. This may be explained by the low number of patients in this subset, the moderate number of patients with missing follow-up information, and by the slightly lower mean OS of patients with TP53 alteration in our set when compared with literature data [40]. Furthermore, we cannot exclude that chromothripsis may be a phenotypical manifestation, or simply an epiphenomenon, of TP53 alteration. Further tests are needed in patients' sets enriched to have TP53 alteration or to have chromothripsis without TP53 alteration. chromosome resulted from chromothripsis of chromosome 11. c With whole chromosome paint probe for chromosome 12 marked in blue revealing part of chromosome 12 on the marker chromosome and on the derivative chromosome 3 from translocation $\mathrm{t}(3 ; 12)$. d Showing deletion of TP53 gene (red signal) in the case with chromothripsis of chromosome 17 annotated as ring chromosome by CBA

Our work describes chromothripsis biological scenario based on SNP high-throughput genomic analyses; even with the limitations due to lack in availability of whole-genome sequencing or gene expression data in our patients' set, we found several events significantly associated with chromothripsis. Patients with chromothripsis presented a high genomic instability, highlighted by the high number of CNA per patient and a high recurrence of losses in chromosome 3 and $5 \mathrm{q}$ and high incidence of complex karyotype; this result is consistent with literature $[9,15,18,24$, 25, 42-44].

Interestingly, we found in most patients with chromothripsis a minimal common-deleted region in 5q31.1-5q33.1, containing key genes involved in RAS, PI3K/AKT, transcriptional factors, DNA damage, histone modification, and SMAD signaling. Moreover, we found heterozygous and homozygous genes' deletions associated with chromothripsis that clustered in relatively small genomic regions. Within altered genes, we hypothesized that FANCA could be a candidate to cooperate at a multigenic and multi-step mechanism that initiate and maintain chromothripsis. FANCA deletion is found in a relatively small genome region; furthermore, it was found deleted in sporadic AML [45] and it originate a syndrome that predispose to AML. Significantly, when we performed pathway enrichment, we found DNA damage and fanconi anemia pathways scoring within the best $1 \%$, together with 
early G0-G1 regulation, cell cycle, and several other pathways that could be possibly related genesis and maintenance of chromothripsis. Tumors characterized by genetic instability and by alterations in DNA damage pathway could be the ideal target of innovative therapeutic approaches like checkpoints inhibitors [46], and combination therapies based on these agents could be an option in chromothripsis patient for patients with poor prognosis. Furthermore, our findings characterize a subset of AML patients with a high burden of alterations and potentially neoantigens that could be the optimal candidates for novel therapies like PD1/PDL1 blocking monoclonal antibodies and immune modulating drugs, maybe in combinations with hypomethylating agents.

Compared to the subset of patients with TP53 alteration, we did not find significant pathways and genes. This may be due to the low number of patients in this subset, and it requires further studies in enriched population with multiomics approach.

Finally, although only a subset of cases could be analyzed by CBA and FISH, our results showed that chromothripsis was associated with marker, derivative, and ring chromosomes, suggesting that these complex chromosomal rearrangements can arise from chromothripsis. This finding is in accordance with what Bochtler and colleagues previously reported [15].

In conclusion, chromothripsis is clearly a catastrophic event defining a consistent group of patients with poor prognosis, which could be candidate per se to novel approaches; chromothripsis is associated with losses of $5 \mathrm{q} 31.1-5 \mathrm{q} 33.1$ in most patients, and with a complex genomic background in which FANCA, TP53, and genes regulating cell cycle seem to be fundamental and demand further preclinical studies.

Acknowledgements Prof. Michele Baccarani and Prof. Michele Cavo directed Istituto Seràgnoli. Prof. Sami Nimer Malek granted access to his previously published data and gave us detailed and useful information on data organization. RK received funding form the Austrian Science Fund (SFB F4702). This work was supported in part by "Progetto Regione-Università 2010-12", by "FP7 NGS-PTL project (agreement no. 306242-NGS-PTL)," and by HARMONY Project. We thank all the members of the NGS-PTL consortium and in particular Prof. Clelia Tiziana Storlazzi. We also thank ELN, AIL, AIRC, and PRIN.

\section{Compliance with ethical standards}

Conflict of interest GM: ARIAD/INCYTE, Pfizer, Celgene, Amgen, $\mathrm{J} \& \mathrm{~J}$, and Roche as consultant. The remaining authors declare that they have no conflict of interest.

Open Access This article is licensed under a Creative Commons Attribution-NonCommercial-NoDerivatives 4.0 International License, which permits any non-commercial use, sharing, distribution and reproduction in any medium or format, as long as you give appropriate credit to the original author(s) and the source, and provide a link to the Creative Commons license. You do not have permission under this license to share adapted material derived from this article or parts of it. The images or other third party material in this article are included in the article's Creative Commons license, unless indicated otherwise in a credit line to the material. If material is not included in the article's Creative Commons license and your intended use is not permitted by statutory regulation or exceeds the permitted use, you will need to obtain permission directly from the copyright holder. To view a copy of this license, visit http://creativecommons.org/licenses/by-nc-nd/4.0/.

\section{References}

1. Byrd JC, Mrózek K, Dodge RK, Carroll AJ, Edwards CG, Arthur DC, et al. Pretreatment cytogenetic abnormalities are predictive of induction success, cumulative incidence of relapse, and overall survival in adult patients with de novo acute myeloid leukemia: results from Cancer and Leukemia Group B (CALGB 8461). Blood. 2002;100:4325-36.

2. Schoch C, Kern W, Kohlmann A, Hiddemann W, Schnittger S, Haferlach T. Acute myeloid leukemia with a complex aberrant karyotype is a distinct biological entity characterized by genomic imbalances and a specific gene expression profile. Genes Chromosomes Cancer. 2005;43:227-38.

3. Döhner H, Estey E, Grimwade D, Amadori S, Appelbaum FR, Büchner T, et al. Diagnosis and management of AML in adults: 2017 ELN recommendations from an international expert panel. Blood. 2017;129:424-47.

4. Meyerson M, Pellman D. Cancer genomes evolve by pulverizing single chromosomes. Cell. 2011;144:9-10.

5. Liu P, Erez A, Nagamani SCS, Dhar SU, Kolodziejska KE, Dharmadhikari AV, et al. Chromosome catastrophes involve replication mechanisms generating complex genomic rearrangements. Cell. 2011;146:889-903.

6. Boeva V, Jouannet $S$, Daveau R, Combaret V, Pierre-Eugène $C$, Cazes A, et al. Breakpoint features of genomic rearrangements in neuroblastoma with unbalanced translocations and chromothripsis. PLoS One. 2013;8:e72182.

7. Tapia-Laliena MA, Korzeniewski N, Hohenfellner M, Duensing S. High-risk prostate cancer: a disease of genomic instability. Urol Oncol. 2014;32:1101-7.

8. Furgason JM, Koncar RF, Michelhaugh SK, Sarkar FH, Mittal S, Sloan $\mathrm{AE}$, et al. Whole genome sequence analysis links chromothripsis to EGFR, MDM2, MDM4, and CDK4 amplification in glioblastoma. Oncoscience. 2015;2:618-28.

9. Rausch T, Jones DTW, Zapatka M, Stütz AM, Zichner T, Weischenfeldt $\mathrm{J}$, et al. Genome sequencing of pediatric medulloblastoma links catastrophic DNA rearrangements with TP53 mutations. Cell. 2012;148:59-71.

10. Kloosterman WP, Koster J, Molenaar JJ. Prevalence and clinical implications of chromothripsis in cancer genomes. Curr Opin Oncol. 2014;26:64-72.

11. Nones K, Waddell N, Wayte N, Patch A-M, Bailey P, Newell F, et al. Genomic catastrophes frequently arise in esophageal adenocarcinoma and drive tumorigenesis. Nat Commun. 2014;5:5224.

12. Przybytkowski E, Lenkiewicz E, Barrett MT, Klein K, Nabavi S, Greenwood CMT, et al. Chromosome-breakage genomic instability and chromothripsis in breast cancer. BMC Genom. 2014;15:579.

13. Hirsch D, Kemmerling R, Davis S, Camps J, Meltzer PS, Ried T, et al. Chromothripsis and focal copy number alterations determine poor outcome in malignant melanoma. Cancer Res. 2013;73:1454-60. 
14. Magrangeas F, Avet-Loiseau H, Munshi NC, Minvielle S. Chromothripsis identifies a rare and aggressive entity among newly diagnosed multiple myeloma patients. Blood. 2011;118:675-8.

15. Bochtler T, Granzow M, Stölzel F, Kunz C, Mohr B, Kartal-Kaess $\mathrm{M}$, et al. Marker chromosomes can arise from chromothripsis and predict adverse prognosis in acute myeloid leukemia. Blood. 2017;129:1333-42.

16. Mackinnon RN, Campbell LJ. Chromothripsis under the microscope: a cytogenetic perspective of two cases of AML with catastrophic chromosome rearrangement. Cancer Genet. 2013;206:238-51.

17. Forero-Castro M, Robledo C, Benito R, Abáigar M, África Martín A, Arefi M, et al. Genome-wide DNA copy number analysis of acute lymphoblastic leukemia identifies new genetic markers associated with clinical outcome. PLoS One. 2016;11: $\mathrm{e} 0148972$.

18. Ratnaparkhe M, Hlevnjak M, Kolb T, Jauch A, Maass KK, Devens F, et al. Genomic profiling of acute lymphoblastic leukemia in ataxia telangiectasia patients reveals tight link between ATM mutations and chromothripsis. Leukemia. 2017;31:2048-56. https://doi.org/10.1038/leu.2017.55.

19. Bassaganyas L, Bea S, Escaramis G, Tornador C, Salaverria I, Zapata L, et al. Sporadic and reversible chromothripsis in chronic lymphocytic leukemia revealed by longitudinal genomic analysis. Leukemia. 2013;27:2376-9.

20. Bertelsen B, Nazaryan-Petersen L, Sun W, Mehrjouy MM, Xie G, Chen W, et al. A germline chromothripsis event stably segregating in 11 individuals through three generations. Genet Med. 2015;18:494-500. https://doi.org/10.1038/gim.2015.112.

21. Cai H, Kumar N, Bagheri HC, von Mering C, Robinson MD, Baudis M. Chromothripsis-like patterns are recurring but heterogeneously distributed features in a survey of 22,347 cancer genome screens. BMC Genom. 2014;15:82.

22. Donley N, Thayer MJ. DNA replication timing, genome stability and cancer: late and/or delayed DNA replication timing is associated with increased genomic instability. Semin Cancer Biol. 2013;23:80-9.

23. Liu G, Stevens JB, Horne SD, Abdallah BY, Ye KJ, Bremer SW, et al. Genome chaos: survival strategy during crisis. Cell Cycle. 2014;13:528-37.

24. Mardin BR, Drainas AP, Waszak SM, Weischenfeldt J, Isokane M, Stütz AM, et al. A cell-based model system links chromothripsis with hyperploidy. Mol Syst Biol. 2015;11:828.

25. Maciejowski J, Li Y, Bosco N, Campbell PJ, de Lange T. Chromothripsis and kataegis induced by telomere crisis. Cell. 2015;163:1641-54.

26. Jacoby MA, De Jesus Pizarro RE, Shao J, Koboldt DC, Fulton RS, Zhou G, et al. The DNA double-strand break response is abnormal in myeloblasts from patients with therapy-related acute myeloid leukemia. Leukemia. 2014;28:1242-51.

27. Govind SK, Zia A, Hennings-Yeomans PH, Watson JD, Fraser M, Anghel C, et al. ShatterProof: operational detection and quantification of chromothripsis. BMC Bioinforma. 2014;15:78.

28. Korbel JO, Campbell PJ. Criteria for inference of chromothripsis in cancer genomes. Cell. 2013;152:1226-36.

29. Stephens PJ, Greenman CD, Fu B, Yang F, Bignell GR, Mudie $\mathrm{LJ}$, et al. Massive genomic rearrangement acquired in a single catastrophic event during cancer development. Cell. 2011;144:27-40.
30. Kovtun IV, Murphy SJ, Johnson SH, Cheville JC, Vasmatzis G. Chromosomal catastrophe is a frequent event in clinically insignificant prostate cancer. Oncotarget. 2015;6:29087-96.

31. Arber DA, Orazi A, Hasserjian R, Thiele J, Borowitz MJ, Le Beau MM, et al. The 2016 revision to the World Health Organization classification of myeloid neoplasms and acute leukemia. Blood. 127:2391-405.

32. World Medical Association. World Medical Association Declaration of Helsinki. JAMA. 2013;310:2191.

33. Harris PA, Taylor R, Thielke R, Payne J, Gonzalez N, Conde JG. Research electronic data capture (REDCap) - A metadata-driven methodology and workflow process for providing translational research informatics support. J Biomed Inform. 2009;42:377-81.

34. Parkin B, Erba H, Ouillette P, Roulston D, Purkayastha A, Karp J, et al. Acquired genomic copy number aberrations and survival in adult acute myelogenous leukemia. Blood. 2010;116:4958-67.

35. R Core Team. R: A language and environment for statistical computing. Vienna, Austria: R Foundation for Statistical Computing; 2016.

36. Fabregat A, Sidiropoulos K, Garapati P, Gillespie M, Hausmann $\mathrm{K}$, Haw R, et al. The reactome pathway knowledgebase. Nucleic Acids Res. 2016;44:D481-7.

37. Estey EH. Acute myeloid leukemia: 2012 update on diagnosis, risk stratification, and management. Am J Hematol. 2012;87:89-99.

38. Krzywinski M, Schein J, Birol I, Connors J, Gascoyne R, Horsman D, et al. Circos: an information aesthetic for comparative genomics. Genome Res. 2009;19:1639-45.

39. Kadia TM, Jain P, Ravandi F, Garcia-Manero G, Andreef M, Takahashi K, et al. TP53 mutations in newly diagnosed acute myeloid leukemia: clinicomolecular characteristics, response to therapy, and outcomes. Cancer. 2016;122:3484-91.

40. Stengel A, Kern W, Haferlach T, Meggendorfer M, Fasan A, Haferlach C. The impact of TP53 mutations and TP53 deletions on survival varies between AML, ALL, MDS and CLL: an analysis of 3307 cases. Leukemia. 2017;31:705-11.

41. Papaemmanuil E, Gerstung M, Bullinger L, Gaidzik VI, Paschka $\mathrm{P}$, Roberts ND, et al. Genomic classification and prognosis in acute myeloid leukemia. N Engl J Med. 2016;374:2209-21.

42. Zhang C-Z, Spektor A, Cornils H, Francis JM, Jackson EK, Liu S, et al. Chromothripsis from DNA damage in micronuclei. Nature. 2015;522:179-84.

43. Leibowitz ML, Zhang C-Z, Pellman D. Chromothripsis: a new mechanism for rapid karyotype evolution. Annu Rev Genet. 2015;49:183-211.

44. Poot M, Haaf T. Mechanisms of origin, phenotypic effects and diagnostic implications of complex chromosome rearrangements. Mol Syndromol. 2015;6:110-34.

45. Tischkowitz MD, Morgan NV, Grimwade D, Eddy C, Ball S, Vorechovsky I, et al. Deletion and reduced expression of the Fanconi anemia FANCA gene in sporadic acute myeloid leukemia. Leukemia. 2004;18:420-5.

46. Ghelli Luserna di Rora' A, Iacobucci I, Martinelli G. The cell cycle checkpoint inhibitors in the treatment of leukemias. J Hematol Oncol. 2017;10:77.

47. Röllig C, Bornhäuser M, Thiede C, Taube F, Kramer M, Mohr B, et al. Long-term prognosis of acute myeloid leukemia according to the new genetic risk classification of the European LeukemiaNet recommendations: evaluation of the proposed reporting system. J Clin Oncol J Am Soc Clin Oncol. 2011;29:2758-65. 